\title{
Impact of Preoperative Serum Tumor Markers in Patients with Lung Squamous Cell Carcinoma
}

\author{
Masaki Tomita*, Shoei Kuroki, Tomoka Hamahiro, Ryo Maeda, Takanori Ayabe \\ Department of Thoracic and Breast Surgery, Faculty of Medicine, University of Miyazaki, Kiyotake, Miyazaki, Japan \\ Email: ^mtomita@med.miyazaki-u.ac.jp
}

How to cite this paper: Tomita, M., Kuroki, S., Hamahiro, T., Maeda, R. and Ayabe, T. (2021) Impact of Preoperative Serum Tumor Markers in Patients with Lung Squamous Cell Carcinoma. Advances in Lung Cancer, 10, 48-55.

https://doi.org/10.4236/alc.2021.103005

Received: August 28, 2021

Accepted: September 6, 2021

Published: September 9, 2021

Copyright (c) 2021 by author(s) and Scientific Research Publishing Inc. This work is licensed under the Creative Commons Attribution International License (CC BY 4.0).

http://creativecommons.org/licenses/by/4.0/

\begin{abstract}
Background: Several previous researchers have investigated the prognostic value of serum tumor markers, especially carcinoembryonic antigen (CEA). Only a limited number of studies reported the usefulness of serum tumor markers for lung squamous cell carcinoma (SQ). We aimed to examine the significance of serum tumor markers for lung SQ. Methods: Eighty-five lung SQ patients who underwent surgery and followed more than 5-year were included. The ratios of 5-year survivors to all patients in groups with several clinicopathologic factors, including tumor markers, were compared. We also compared the clinicopathologic factors between central type and peripheral type SQ. Results: The majority of patients were male gender and current/ former smokers. Age, $\mathrm{pN}$ status, cytokeratin-19 fragment (CYFRA 21-1), squamous cell carcinoma antigen (SCC), and comorbid interstitial pneumonia (IP) were associated with the ratio of 5-year survivors significantly. When patients were compared based on tumor location, high p-stage and CYFRA 21-1 were related to central type SQ. Conclusion: Both SCC and CYFRA 21-1 appeared to be useful prognostic markers for patients with lung SQ. Furthermore, CYFRA 21-1 was related to central type SQ.
\end{abstract}

\section{Keywords}

Lung Squamous Cell Carcinoma, Cytokeratin-19 Fragment, Squamous Cell Carcinoma Antigen

\section{Introduction}

Serum tumor markers have been investigated as prognostic factors for non-small cell lung cancer (NSCLC). Preoperative carcinoembryonic antigen (CEA) has emerged as an independent prognostic factor for patients with NSCLC, especially adenocarcinoma (AD) [1] [2] [3]. Although several studies reported the 
prognostic role of CEA [1] [2] [3], only a limited number of recent previous studies reported the prognostic role of squamous cell carcinoma antigen (SCC) in NSCLC [2] [4] [5] [6]. Emerging evidence suggests that preoperative cytokeratin-19 fragment (CYFRA 21-1) is now known as a useful tumor marker for NSCLC [2] [3] [7] [8]. Both SCC and CYFRA 21-1 are generally known to be sensitive to squamous cell carcinoma (SQ) as compared to $\mathrm{AD}$ [2] [4] [5] [7] [8]. However, the majority of previous studies examined all NSCLC patients, including $\mathrm{AD}$ and $\mathrm{SQ}$. The number of previous studies which focused on lung SQ is small, although SQ is one of the major NSCLC [2] [7] [8]. Therefore, in the present study, the prognostic usefulness of preoperative tumor markers for lung SQ was investigated.

Furthermore, lung SQ can be classified into a central type (c-SQ) and a peripheral type (p-SQ) based on the tumor location [9] [10]. Recently, it has been well accepted that c-SQ and p-SQ have different clinicopathologic characteristics and should be classified into different categories [9] [10]. Therefore, we also compared the clinical factors, including tumor markers, between c-SQ and p-SQ.

\section{Patients and Methods}

The Medical Ethics Committee of our hospital approved the study protocol (O-0997) and waiver of the written informed consents from each patient, considering the retrospective design and anonymous clinical data. A total of 684 patients with newly diagnosed NSCLC who received curative resections at our hospital over a 10-year period up to June 2016, were retrospectively analyzed. Patients with loss to follow-up within 5 years were excluded from this study. All patients were followed for more than 5 years. Among these patients, there are 85 patients with primary lung SQ.

Preoperative complete blood count and routine biochemistry test, including serum CEA, SCC, and CYFRA 21-1, of each patient, was applied within 1 month before surgery. Upper cutoff levels of serum CEA, SCC, and CYFRA 21-1 were $5.0 \mathrm{ng} / \mathrm{ml}, 1.5 \mathrm{ng} / \mathrm{ml}$, and $2.3 \mathrm{ng} / \mathrm{ml}$, respectively. Preoperative data were retrospectively extracted from the patients' medical records. All patients in this study were staged according to the $8^{\text {th }}$ edition Cancer Staging [11].

For survival analysis, Sagawa et al. [12] reported that the calculated cumulative survival rate occasionally can be confounded by those patients with a short follow-up period. Therefore, they presented the clinicopathologic characteristics of long-term survivors using a follow-up period of more than 5 years and an actual number of survivors [12]. According to this manuscript, in the present study, we omitted the patients with a follow-up period of fewer than 5 years and analyzed the actual number of survivors rather than the cumulative survival rate. Pearson's chi-square analysis with Yate's correction was used to compare the various rates. A p-values $<0.05$ was considered statistically significant. All statistical analyses were performed using JMP Pro software, version 15 (SAS Institute, Cary, NC, USA). 


\section{Results}

The majority of our lung SQ patients were male gender (76/85) and current/ former smokers (78/85). There were 40 patients with c-SQ and 45 with p-SQ. Forty-one patients (48.24\%) survived more than 5 years.

As shown in Table 1, we compared the 5-year survivors and others based on the various clinicopathologic factors. Our result showed that age, $\mathrm{pN}$ status, and comorbid interstitial pneumonia (IP) were associated with the ratio of 5-year survivors, whereas, gender, smoking status, $\mathrm{pT}$ status, and tumor location were not. The p-stage was marginally significantly associated with 5-year survivors ( $p$ $=0.054)$.

Concerning serum tumor markers, we failed to find the difference in CEA

Table 1. Comparison between 5-year survivors and others.

\begin{tabular}{|c|c|c|c|c|}
\hline & & 5-year survivors & Non-survivors & $p$ Value \\
\hline \multirow[t]{2}{*}{ Age } & $<65$ & 15 & 7 & 0.028 \\
\hline & $\geq 65$ & 26 & 37 & \\
\hline \multirow[t]{2}{*}{ Gender } & Male & 35 & 41 & 0.239 \\
\hline & Female & 6 & 3 & \\
\hline \multirow[t]{2}{*}{ Smoking status } & Current/former & 39 & 39 & 0.269 \\
\hline & Never & 2 & 5 & \\
\hline \multirow[t]{2}{*}{ p-stage } & I & 28 & 21 & 0.054 \\
\hline & II-III & 13 & 23 & \\
\hline \multirow[t]{2}{*}{ pT status } & pT1 & 22 & 16 & 0.108 \\
\hline & pT2-3 & 19 & 28 & \\
\hline \multirow[t]{2}{*}{$\mathrm{pN}$ status } & pNo & 35 & 27 & 0.011 \\
\hline & $\mathrm{pN} 1-2$ & 6 & 17 & \\
\hline \multirow[t]{2}{*}{ Tumor location } & Central & 19 & 21 & 0.898 \\
\hline & Peripheral & 22 & 23 & \\
\hline \multirow[t]{2}{*}{ IP } & $\mathrm{IP}+$ & 3 & 10 & 0.043 \\
\hline & IP- & 38 & 34 & \\
\hline \multirow[t]{2}{*}{ CEA } & Normal & 30 & 31 & 0.781 \\
\hline & High & 11 & 13 & \\
\hline \multirow[t]{2}{*}{ SCC } & Normal & 28 & 16 & 0.003 \\
\hline & High & 13 & 28 & \\
\hline \multirow[t]{2}{*}{ CYFRA 21-1 } & Normal & 29 & 18 & 0.005 \\
\hline & High & 12 & 26 & \\
\hline \multirow[t]{2}{*}{ SCC \& CYFRA 21-1 } & Both normal & 23 & 9 & 0.001 \\
\hline & Others & 18 & 35 & \\
\hline
\end{tabular}

IP: Interstitial pneumonia; CEA: Carcinoembryonic antigen; SCC: Squamous cell carcinoma antigen; CYFRA 21-1: Cytokeratin-19 fragment. 
between 5-year survivors and others. However, 28/44 (63.6\%) patients with normal SCC were 5-year survivors, whereas 13/41 (31.7\%) with high SCC survived more than 5 years $(p=0.003)$. Similarly, $29 / 47$ patients $(61.7 \%)$ with normal CYFRA 21-1 were 5-year survivors, whereas 12/38 (31.6\%) with high CYFRA 21-1 survived $(p=0.005)$. The relationship between 5 -year survivors and serum tumor markers had been also shown as a box plot with a scatter diagram (Figure 1). To examine the combined use of tumor markers, SQ patients were subdivided into 2 groups based on SCC and CYFRA 21-1; both normal group and others. Twenty-three of 32 (71.9\%) patients with both normal were 5 -year survivors, whereas 18/53 (34.0\%) with others survived more than 5 years $(p=0.001)$.

Although the ratio of 5-year survivors was not different between c-SQ and p-SQ, the clinical characteristics were compared between c-SQ and p-SQ (Table 2). Age, gender, smoking status, $\mathrm{pT}$ status, $\mathrm{pN}$ status, CEA, and SCC were not related to tumor location. There was a trend towards an association between p-SQ and comorbid IP, but this did not reach statistical significance $(p=0.053)$. On the other hand, high $\mathrm{p}$-stage was significantly related to c-SQ $(p=0.026)$. Furthermore, 16/47 (34.0\%) patients with normal CYFRA 21-1 were c-SQ, whereas 24/38 (63.2\%) with high CYFRA 21-1 were c-SQ ( $p=0.007)$. Figure 2 also showed a box plot with a scatter diagram comparing the c-SQ and p-SQ based on tumor markers.

(a)

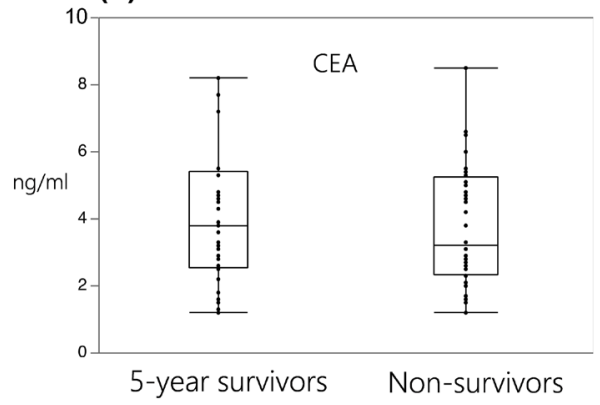

(b)

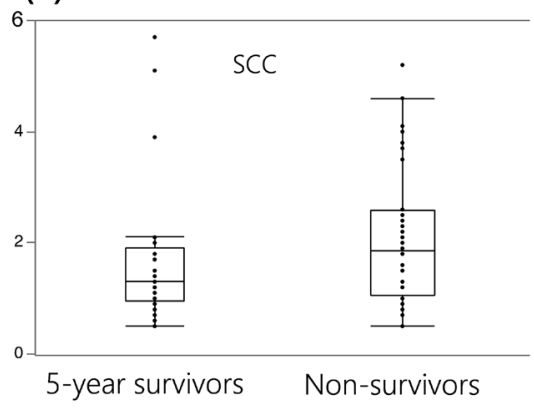

(c)

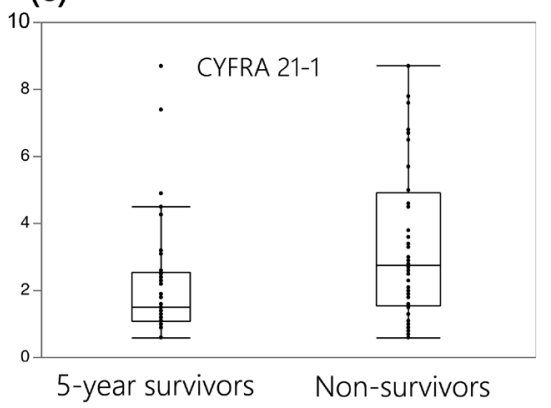

Figure 1. A box plot with a scatter diagram comparing the 5-year survivors and others based on CEA (a), SCC (b), and CYFRA 21-1 (c).

(a)

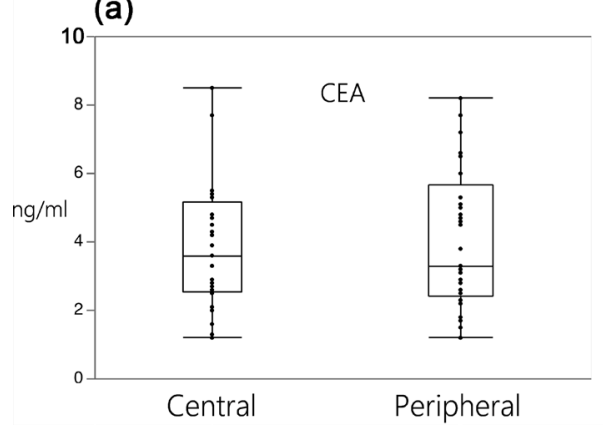

(b)

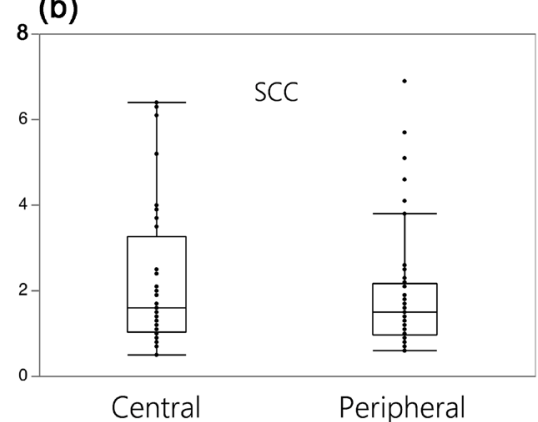

(c)

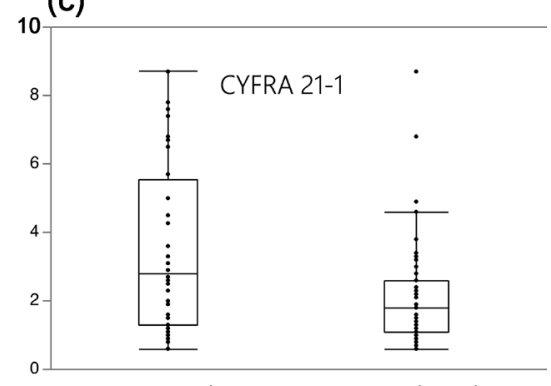

Central
Peripheral

Figure 2. A box plot with a scatter diagram comparing the central and peripheral type based on CEA (a), SCC (b), and CYFRA 21-1 (c). 
Table 2. Comparison between c-SQ and p-SQ.

\begin{tabular}{|c|c|c|c|c|}
\hline & & c-SQ & p-SQ & $p$ Value \\
\hline \multirow[t]{2}{*}{ Age } & $<65$ & 10 & 12 & 0.861 \\
\hline & $\geq 65$ & 30 & 33 & \\
\hline \multirow[t]{2}{*}{ Gender } & Male & 37 & 39 & 0.378 \\
\hline & Female & 3 & 6 & \\
\hline \multirow[t]{2}{*}{ Smoking status } & Current/former & 36 & 42 & 0.577 \\
\hline & Never & 4 & 3 & \\
\hline \multirow[t]{2}{*}{ p-stage } & I & 18 & 31 & 0.026 \\
\hline & II-III & 22 & 14 & \\
\hline \multirow{2}{*}{ pT status } & pT1 & 15 & 23 & 0.207 \\
\hline & pT2-3 & 25 & 22 & \\
\hline \multirow[t]{2}{*}{$\mathrm{pN}$ status } & $\mathrm{pN} 0$ & 26 & 36 & 0.120 \\
\hline & $\mathrm{pN} 1-2$ & 14 & 9 & \\
\hline \multirow[t]{2}{*}{ IP } & $\mathrm{IP}+$ & 3 & 10 & 0.053 \\
\hline & IP- & 37 & 35 & \\
\hline \multirow[t]{2}{*}{ CEA } & Normal & 30 & 31 & 0.531 \\
\hline & High & 10 & 14 & \\
\hline \multirow[t]{2}{*}{ SCC } & Normal & 19 & 25 & 0.458 \\
\hline & High & 21 & 20 & \\
\hline \multirow[t]{2}{*}{ CYFRA 21-1 } & Normal & 16 & 31 & 0.007 \\
\hline & High & 24 & 14 & \\
\hline
\end{tabular}

c-SQ: Central type squamous cell carcinoma; p-SQ: Peripheral type squamous cell carcinoma; IP: Interstitial pneumonia; CEA: Carcinoembryonic Antigen; SCC: Squamous Cell Carcinoma antigen; CYFRA 21-1: Cytokeratin-19 fragment.

\section{Discussion}

The SCC is a widely used tumor marker for NSCLC, especially for SQ. The prognosis of patients with squamous cell carcinomas originating from the head and neck, and esophagus had been shown to be associated with SCC [13] [14]. However, in manuscripts published in the $21^{\text {st }}$ century, only a limited number of manuscripts report the prognostic significance of SCC in NSCLC [2] [4] [5] [6] [7] and the results were conflicting. Kinoshita et al. [2] showed that high SCC or vascular invasion were independent prognostic factors in lung SQ, and they proposed that the current stage IA SQ with high SCC or vascular invasion should be upgraded to stage IB. Yu et al. [4] found that the overall survival was significantly shorter in lung SQ patients with high SCC. On the other hand, Takeuchi et al. [5] concluded that preoperative SCC did not reflect the prognosis in lung SQ, although they found that the survival rate was higher in high SCC patients who became normal SCC following resection than in patients who remained high SCC. Vassilakopoulos et al. [6] failed to find the prognostic roles of 
preoperative SCC, although they included not only SQ, but also other NSCLCs. Kulpa et al. [7] also revealed that SCC was not an independent prognostic factor in lung SQ patients. Our result showed preoperative SCC was related to 5-year survivors in lung SQ. These conflicting findings might be due to a small number of patients, and differences in cut-off values of each study. Further, there might be a possibility that the prognostic power of SCC is limited in lung SQ.

In contrast to SCC, several studies reported the prognostic value of CYFRA 21-1 in NSCLC [2] [3] [7] [8]. Although CYFRA 21-1 is generally known to be sensitive to SQ, there are only a limited number of previous studies that focused on SQ [7] [8]. Kulpa et al. [7] revealed that CYFRA 21-1 was an independent prognostic factor in the operable patients with lung SQ. Niklinski et al. [8] also obtained a relationship between CYFRA 21-1 and prognosis in patients with lung SQ. The present study also showed that preoperative CYFRA 21-1 was also related to 5-year survivors. Although the number of previous studies for CYFRA 21-1 focused on lung SQ is small, to our knowledge, there are no studies that failed to find the prognostic value of CYFRA 21-1. Our result also showed the possible utility of tumor marker combination with SCC and CYFRA 21-1. Kashiwabara et al. [15] also examined the utility of tumor marker combination and showed that the prognosis of the high levels of both SCC and CYFRA 21-1 group was significantly lower. However, they also found no prognostic differences between the high SCC/normal CYFRA 21-1 group and both normal group and between the normal SCC/high CYFRA group and both high groups [15]. They concluded that CYFRA 21-1, not SCC, relates to the prognosis [15]. Unfortunately, they did not show a prognostic significance of either SCC or CYFRA 21-1 alone. Furthermore, 63/120 their patients were non-surgery patients, and the prognosis was examined using the 2-year survival rates [15].

Based on tumor location, lung SQ can be classified into c-SQ and p-SQ [9] [15]. Not only tumor location, but also the clinicopathologic characteristics of these have been known to be different [9] [15]. Lin et al. [9] summarized the previous studies that investigated the clinicopathologic differences between c-SQ and $\mathrm{p}$-SQ. They concluded that the patients with $\mathrm{p}-\mathrm{SQ}$ were characterized by older age, less frequent lymph node metastasis, an earlier pathologic stage, good differentiation, less lymphovascular invasion, and less pleural invasion [9]. However, the differences in tumor markers between c-SQ and p-SQ have not been reported. Saijo et al. [15] compared the tumor markers between c-SQ and p-SQ and found that CEA, SCC, and neuron-specific enolase were not significantly different. Unfortunately, the differences in CYFRA 21-1 between c-SQ and $\mathrm{p}$-SQ have not been investigated. Our result showed that high p-stage and CYFRA 21-1 were related to c-SQ. To our knowledge, this is the first report that showed differences in CYFRA 21-1 based on different tumor locations of lung SQ. The biological significance of this difference in CYFRA 21-1 between c-SQ and $\mathrm{p}-\mathrm{SQ}$ is not clear and merits further investigation.

Our study had several limitations. It was a single-center, retrospective study 
with relatively small sample size. Thus, conclusions from the present study may have a bias, indicating that studies using a larger population are needed.

\section{Conclusion}

We demonstrate that preoperative SCC and CYFRA 21-1 were related to 5-year survivors in patients with lung SQ. Therefore, both SCC and CYFRA 21-1 appeared to be useful prognostic markers for patients with lung SQ. Furthermore, CYFRA 21-1 was related to central type SQ.

\section{Conflicts of Interest}

The authors declare no conflicts of interest regarding the publication of this paper.

\section{References}

[1] Grunnet, M. and Sorensen, J.B. (2012) Carcinoembryonic Antigen (CEA) as Tumor Marker in Lung Cancer. Lung Cancer, 76, 138-143.

https://doi.org/10.1016/j.lungcan.2011.11.012

[2] Kinoshita, T., Ohtsuka, T., Yotsukura, M., Asakura, K., Goto, T., Kamiyama, I., Otake, S., Tajima, A., Emoto, K., Hayashi, Y. and Kohno, M. (2015) Prognostic Impact of Preoperative Tumor Marker Levels and Lymphovascular Invasion in Pathological Stage I Adenocarcinoma and Squamous Cell Carcinoma of the Lung. Journal of Thoracic Oncology, 10, 619-628. https://doi.org/10.1097/JTO.0000000000000480

[3] Tomita, M., Shimizu, T., Ayabe, T., Yonei, A. and Onitsuka, T. (2010) Prognostic Significance of Tumour Marker Index Based on Preoperative CEA and CYFRA 21-1 in Non-Small Cell Lung Cancer. Anticancer Research, 30, 3099-3102.

[4] Yu, D., Du, K., Liu, T. and Chen, G. (2013) Prognostic Value of Tumor Markers, NSE, CA125 and SCC, in Operable NSCLC Patients. International Journal of Molecular Sciences, 14, 11145-11156. https://doi.org/10.3390/ijms140611145

[5] Takeuchi, S., Nonaka, M., Kadokura, M. and Takaba, T. (2003) Prognostic Significance of Serum Squamous Cell Carcinoma Antigen in Surgically Treated Lung Cancer. Annals of Thoracic and Cardiovascular Surgery, 9, 98-104.

[6] Vassilakopoulos, T., Troupis, T., Sotiropoulou, C., Zacharatos, P., Katsaounou, P., Parthenis, D., Noussia, O., Troupis, G., Papiris, S., Kittas, C., Roussos, C., Zakynthinos, S. and Gorgoulis, V. (2001) Diagnostic and Prognostic Significance of Squamous Cell Carcinoma Antigen in Non-Small Cell Lung Cancer. Lung Cancer, 32, 137-144. https://doi.org/10.1016/S0169-5002(00)00225-7

[7] Kulpa, J., Wójcik, E., Reinfuss, M. and Kołodziejski, L. (2002) Carcinoembryonic Antigen, Squamous Cell Carcinoma Antigen, CYFRA 21-1, and Neuron-Specific Enolase in Squamous Cell Lung Cancer Patients. Clinical Chemistry, 48, 1931-1937. https://doi.org/10.1093/clinchem/48.11.1931

[8] Niklinski, J., Furman, M., Chyczewska, E., Chyczewski, L., Rogowski, F. and Laudanski, J. (1995) Diagnostic and Prognostic Value of the New Tumour Marker CYFRA 21-1 in Patients with Squamous Cell Lung Cancer. The European Respiratory Journal, 8, 291-294. https://doi.org/10.1183/09031936.95.08020291

[9] Lin, M.W., Huang, Y.L., Yang, C.Y., Kuo, S.W., Wu, C.T. and Chang, Y.L. (2019) The Differences in Clinicopathologic and Prognostic Characteristics between Surgically Resected Peripheral and Central Lung Squamous Cell Carcinoma. Annals of 
Surgical Oncology, 26, 217-229. https://doi.org/10.1245/s10434-018-6993-5

[10] Saijo, T., Ishii, G., Nagai, K., Funai, K., Nitadori, J., Tsuta, K., Nara, M., Hishida, T. and Ochiai, A. (2006) Differences in Clinicopathological and Biological Features between Central-Type and Peripheral-Type Squamous Cell Carcinoma of the Lung. Lung Cancer, 52, 37-45. https://doi.org/10.1016/j.lungcan.2005.12.006

[11] Goldstraw, P., Chansky, K., Crowley, J., Rami-Porta, R., Asamura, H., Eberhardt, W.E., Nicholson, A.G., Groome, P., Mitchell, A. and Bolejack, V. (2016) The IASLC Lung Cancer Staging Project: Proposals for Revision of the TNM Stage Groupings in the Forthcoming (Eighth) Edition of the TNM Classification for Lung Cancer. Journal of Thoracic Oncology, 11, 39-51. https://doi.org/10.1016/j.jtho.2015.09.009

[12] Sagawa, M., Sakurada, A., Fujimura, S., Sato, M., Takahashi, S., Usuda, K., Endo, C., Aikawa, H., Kondo, T. and Saito, Y. (1999) Five-Year Survivors with Resected pN2 Nonsmall Cell Lung Carcinoma. Cancer, 85, 864-868.

https://doi.org/10.1002/(SICI)1097-0142(19990215)85:4<864::AID-CNCR13>3.0.C $\underline{\mathrm{O} ; 2-\mathrm{Q}}$

[13] Imai, R., Takenaka, Y., Yasui, T., Nakahara, S., Yamamoto, Y., Hanamoto, A., Takemoto, N., Fukusumi, T., Cho, H., Yamamoto, M. and Inohara, H. (2015) Prognostic Significance of Serum Squamous Cell Carcinoma Antigen in Patients with Head and Neck Cancer. Acta Oto-Laryngologica, 135, 295-301.

https://doi.org/10.3109/00016489.2014.951454

[14] Kanda, M., Koike, M., Shimizu, D., Tanaka, C., Kobayashi, D., Hattori, N., Hayashi, M., Omae, K., Yamada, S., Nakayama, G. and Kodera, Y. (2020) Optimized Cutoff Value of Serum Squamous Cell Carcinoma Antigen Concentration Accurately Predicts Recurrence After Curative Resection of Squamous Cell Carcinoma of the Esophagus. Annals of Surgical Oncology, 27, 1233-1240. https://doi.org/10.1245/s10434-019-07977-6

[15] Kashiwabara, K., Nakamura, H. and Esaki, T. (2000) Prognosis in Bronchogenic Squamous Cell Carcinoma Groups Divided According to Serum Squamous Cell Carcinoma-Related Antigen and Cytokeratin 19 Fragment Levels. Clinica Chimica Acta, 294, 105-113. https://doi.org/10.1016/S0009-8981(99)00247-8 